\title{
Standardized Mixed Language Programming for Fortran and $\mathrm{C}$
}

\author{
Bo Einarsson, Richard J. Hanson†, Tim Hopkins ${ }^{\ddagger}$
}

March 6, 2010

\section{Introduction}

At a conference in Kyoto 1995 a lecture about the use of routines in $\mathrm{C}$ from Fortran, and the opposite, was presented (Einarsson, 1995). At that time, it was then necessary to describe this differently for each different platform.

Fortran 2003 has standardized the mixing of Fortran and C with the concept of "Interoperability with C." Many Fortran compilers already implement parts of the 2003 standard, see (Chivers and Sleightholme, 2009). We look at a number of different aspects of mixed language programming and present examples to demonstrate the facilities that are available in the new standard.

We have checked all these examples using the following compilers

1. Intel Fortran compiler 10.1,

2. IBM XL Fortran Enterprise Edition for AIX, V11.1 (5724-S72) Version 11.01.0000.0001D, (September 19, 2007),

3. Sun Fortran 958.3 (July 18, 2007),

4. NAGWare Fortran 95 compiler Release 5.2 (668),

5. g95 version 0.92 (March 14, 2009),

6. gfortran version 4.4.0 (February 19, 2009), and

7. The Portland Group, pgf90 8.0-5 64-bit target on x86-64 Linux.

There is an old recommendation for mixed language programming (or inter-language communication) that only one of the languages should be used for input and output. We violate this recommendation in the last example of Section 4, where output is generated both by Fortran and C. The order of the output may differ on different platforms due to the fact that both Fortran and $\mathrm{C}$ may buffer output for efficiency reasons. This is especially noticeable when output is redirected to a file. This problem can often be ameliorated by judicious use of the flush statement in both languages.

The 2003 standard allows data to be exchanged between Fortran and C provided that it is represented and interpreted in the same way in each language. The tables given in (Metcalf et al., 2004, Table 14.1) and (Adams et al., 2008, Table 15-1) detail how to choose the correct Fortran kind values to ensure compatibility between the two languages. We restrict the use of these KIND values to the small wrapper routines that we write to implement cross calling.

\footnotetext{
*Mathematics Department, University of Linköping, SE-581 83 Linköping, Sweden. bo.einarsson@liu.se

†Visual Numerics Inc., Home: 13416 Circulo Largo NE, Albuquerque, NM 87112, USA. richard.koolhans@gmail.com

${ }^{\ddagger}$ Computing Laboratory, University of Kent, Canterbury, Kent CT2 7NF, England. T.R.Hopkins@kent.ac.uk
} 
The BIND attribute and the iso_c_binding intrinsic module provide the necessary facilities to write standard conforming code that will allow Fortran and $\mathrm{C}$ components to interoperate. It may also be the case that a Fortran compiler requires a specific $\mathrm{C}$ compiler to be used in order for interoperability to take place.

Note that there may be traps for the unwary when using a Fortran 2003 compatible compiler to compile and link mixed Fortran and $\mathrm{C}$ code that was written to make use of the old platformdependent mechanisms. We have encountered examples where the combination of a new compiler and ad hoc methods cause unexpected run-time errors. Typically these are caused by problems relating to the length of string arguments.

A simplified form of an example that caused such as error is given by these code fragments:

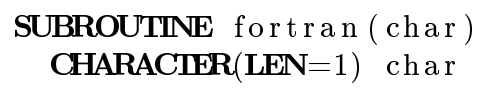

The calling $\mathrm{C}$ routine has no direct language support for specifying the length of the Fortran character argument "L". The Fortran run-time system, if it is "picky," can certainly check that the length of this argument has a value at least one. By chance the value in memory might be positive, but otherwise arbitrary. Or the Fortran system can assume that the length of the character is exactly one and make no check. But if by chance the arbitrary value is non-positive, then the "picky" Fortran system can rightly issue an error. The NAGWare Fortran 95 compiler gives an instance where exactly this error occurs.

Our first example checks to see if the basic data types are compatible between the two languages. With the exception of the Portland compiler ${ }^{1}$, running the code given in Listing 1 gives the following results

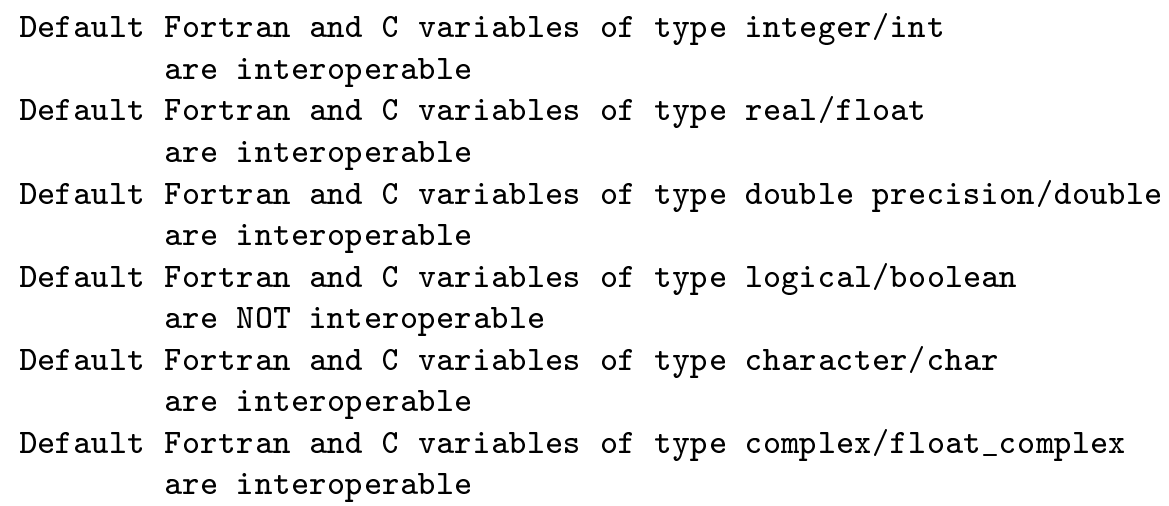

Listing 1: CandF.f90

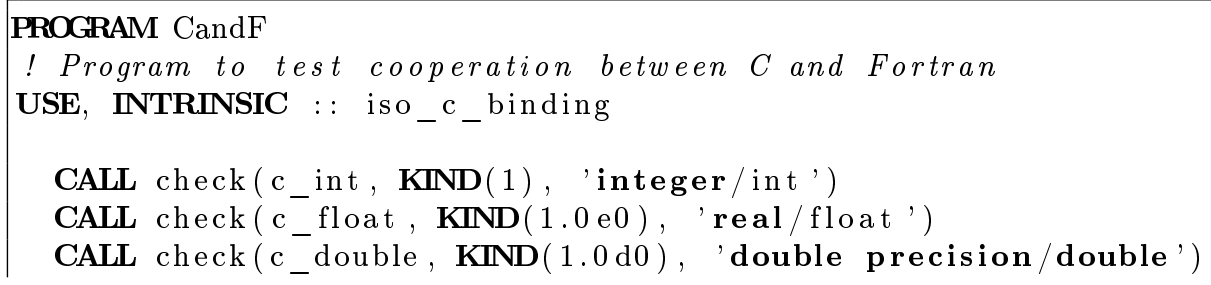

\footnotetext{
${ }^{1}$ The Portland 8.0 compiler gives an error message that a KIND value is negative, indicating a not implemented data type.
} 


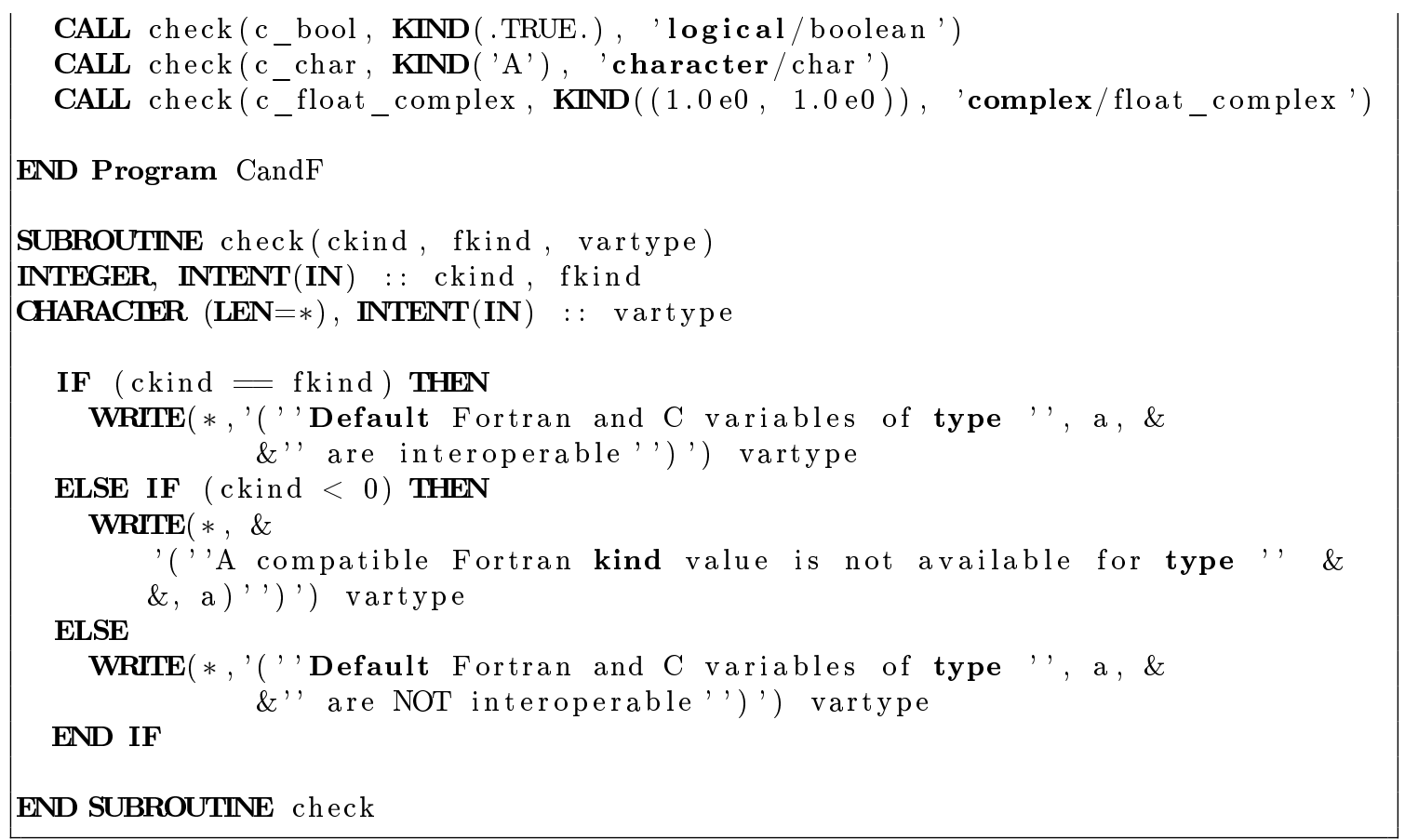

The value returned for $c_{-}$bool indicates that a compatible LOGICAL (KIND=c_bool) is provided by the compiler but this must be explicitly declared as it differs from the KIND value associated with the default LOGICAL. In this instance we can arrange to convert between the Fortran and $\mathrm{C}$ types using intrinsic overloaded assignment in the communication (or wrapper) routine. For example

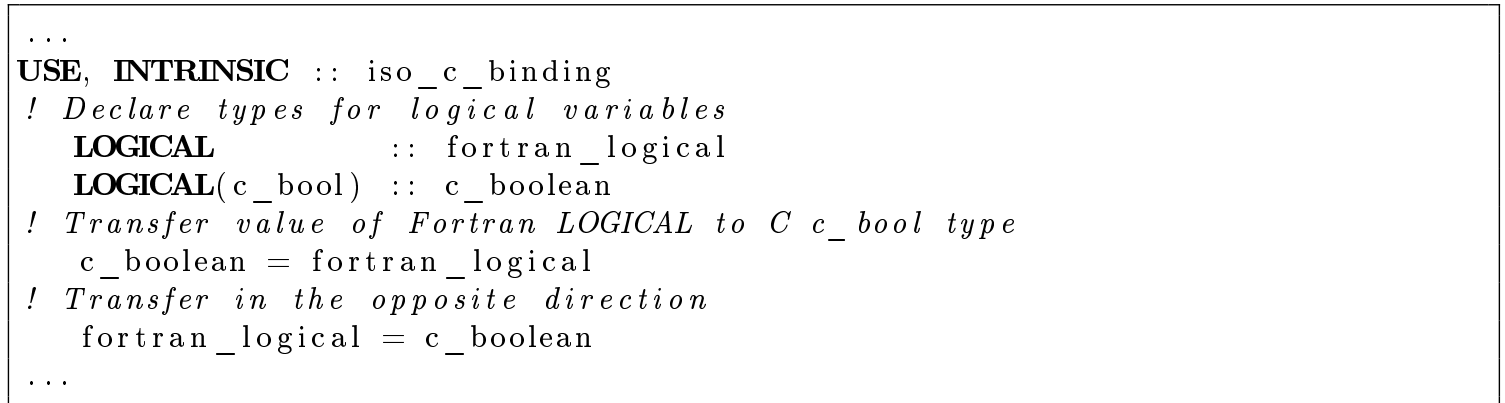

With version 8.0 of the Portland compiler a system dependent solution has to be used. For example a wrapper $\mathrm{C}$ routine can convert a $c_{-}$bool or _ Bool variables to $c_{-}$int variables, with $\{0,1\}$ values, that are communicated to Fortran.

\section{Use of a Fortran subroutine/function from a C program}

We begin by showing what we need to do to call a simple Fortran subroutine and function from C. This illustrates how a wrapper routine can be constructed to allow calls to be made from $\mathrm{C}$ even when the original source code to the Fortran procedures is not available.

We start by defining the two simple Fortran subprograms (Listing 3) and constructing a Fortran driver program (Listing 2) to call them. Running this program results in the output
$6 \quad 648$
Bo G E

Listing 2: f2sam.f90 


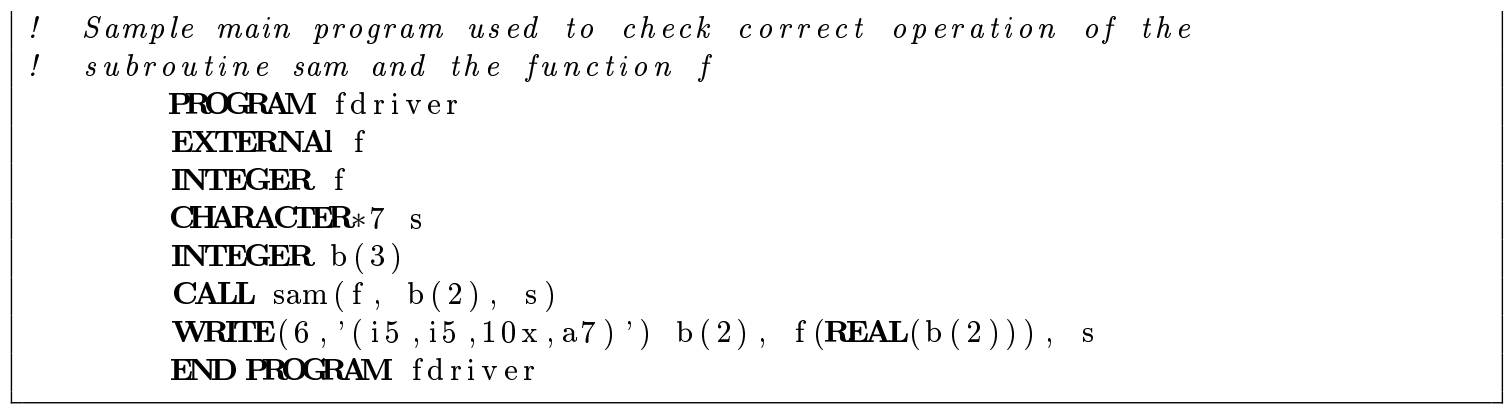

Listing 3: sam.f90

\begin{tabular}{|c|}
\hline $\begin{array}{l}\text { SUBROUTINE } \operatorname{sam}(\mathrm{f}, \mathrm{b}, \mathrm{s}) \\
\text { EXTERNAL f } \\
\text { INTEGER f } \\
\text { INTEGER, INTENT(OUT) : : b } \\
\text { OHARACIER(LEN=7), INTENT(OUT) }:: \mathrm{s} \\
\text { REAL : } \mathrm{x} \\
\mathrm{x}=1.3 \\
\mathrm{~s}=\text { Bo G } \mathrm{E}, \\
\mathrm{b}=\mathrm{f}(\mathrm{x}) \\
\text { END SUBROUTINE sam } \\
\text { INTEGER FUNCTION } \mathrm{f}(\mathrm{x}) \\
\text { REAL, INTENT(IN) : : } \mathrm{x} \\
\mathrm{f}=3 * \mathrm{x} * * 3 \\
\text { REIURN } \\
\text { END FUNCTION } \mathrm{f}\end{array}$ \\
\hline
\end{tabular}

We now look at what needs to be done to make the procedures sam and $f$ callable from $\mathrm{C}$ without the need for any changes to the original code. We achieve this in Listing 4 by constructing wrapper or communication routines in Fortran which call the original procedures.

We draw attention here to two important requirements in the interoperability of character parameters. Only a scalar or an array of CHARACTER (LEN=1) is interoperable although the standard does allow an actual argument of length greater than one to be used when the dummy argument is an array of CHARACTER ( $L E N=1$ ). Second, C expects that strings will be terminated with the null character; failure to do this may result in fatal errors if $\mathrm{C}$ attempts to process the string with, for example, strcpy. This means that a $\mathrm{C}$ string will be one character longer than its equivalent Fortran string.

Listing 4: c sam.f90

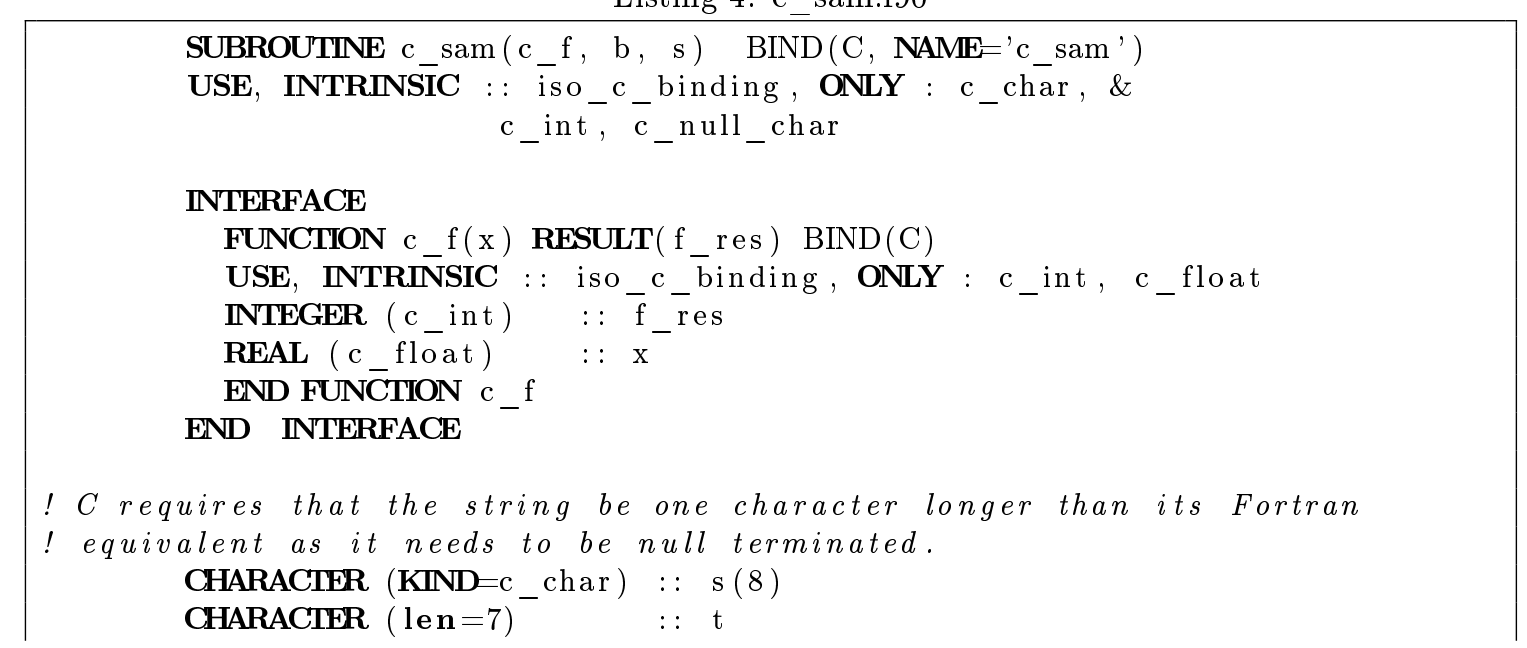




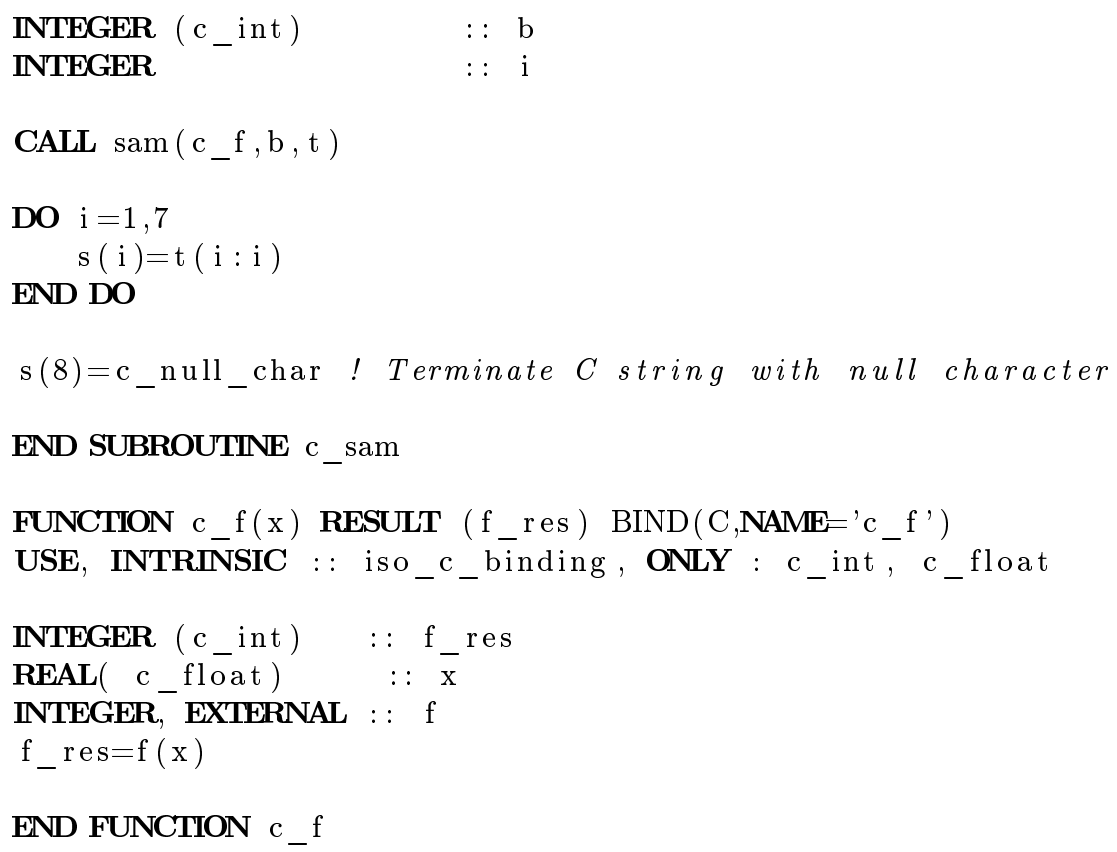

Finally, in Listing 5 we give the $\mathrm{C}$ program that calls the original routines sam and $f$ via the wrapper routines $c_{-}$sam and c_f respectfully. Note that the function prototypes of any Fortran routines called by the $\mathrm{C}$ program must be included.

Listing 5: c2sam.c

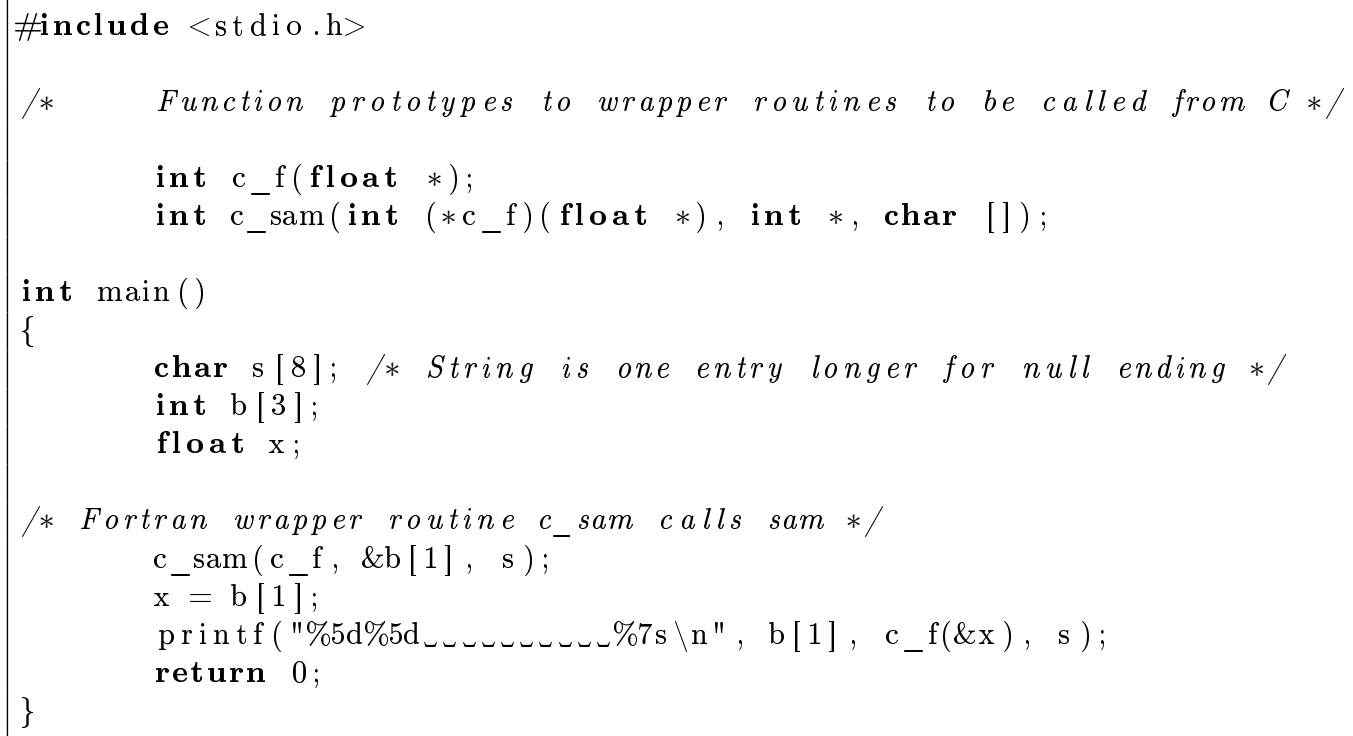

We may compile and run these on a Unix/Linux system using a set of commands of the form

cc -c c2sam.c

f95 c_sam.f90 sam.f90 c2sam.o

a. out

to give the result

$6648 \quad$ Bo G E

as before. 


\section{Use from Fortran of a matrix assigned values in $\mathrm{C}$}

In this case we wish to initialize elements of an array via a function written in $\mathrm{C}$ and use these values in a Fortran program. Because the Fortran standard mandates that array elements are stored in column major order and $\mathrm{C}$ requires their storage in row major order, we need to reverse the order of the indices to ensure that both languages use the same ordering of elements within memory. In addition, we need to remember that in $\mathrm{C}$ an array declared a [3] [2] defines elements $\mathrm{a}[0][0] \ldots a$ [2] [1] and we must adjust our indexing accordingly. The $\mathrm{C}$ routine is given in Listing 6 while the Fortran main program is provided in Listing 7.

Listing 6: mlp4.c

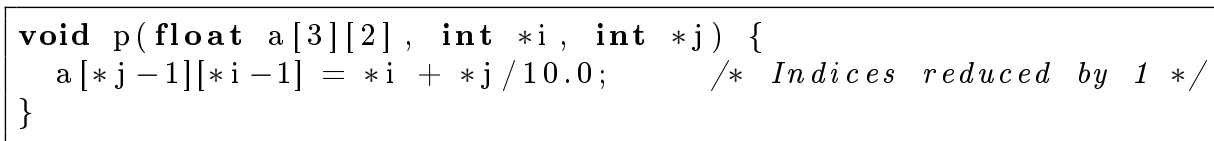

Listing 7: mlp3.f90

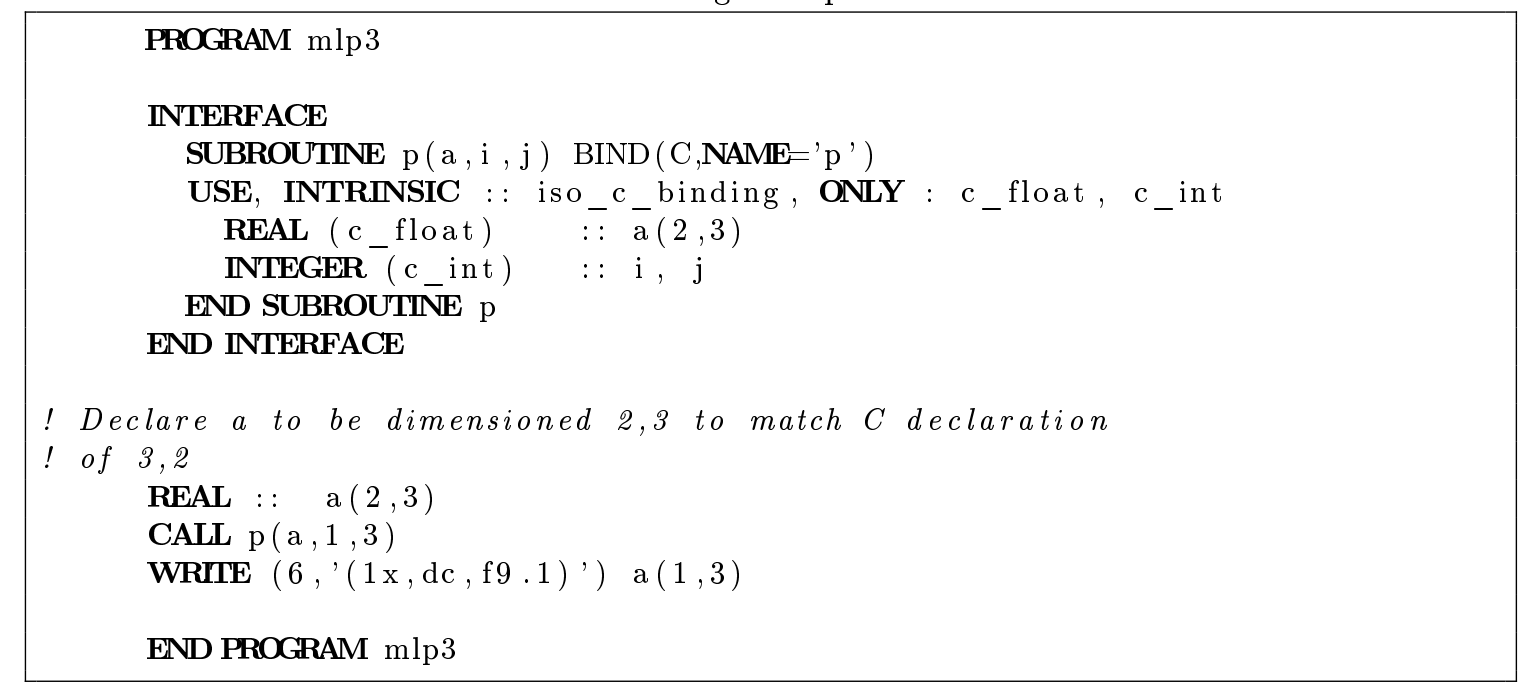

These are run in Unix/Linux with

cc $\quad-c \operatorname{mlp} 4 . c$

f95 mlp3.f90 mlp4.o

a. out

with the result ${ }^{2} 1,3$.

\section{Use of Fortran COMMON data in a $\mathrm{C}$ program}

As in the first example we wish to use a Fortran routine from both Fortran and C, therefore we once again need a communication routine. The COMMON block is assigned its values in the routine init_name (Listing 9) which together with the Fortran driver in Listing 8 give the result 786 3.2.

Listing 8: mlp0.f90

PROGRAM mlp0

INTEGER i

REAL $\mathrm{r}$

COMMON /name/ i , r

\footnotetext{
${ }^{2}$ The Fortran 2003 command dc for decimal comma does not work on the Portland compiler.
} 
CALL init name

$\operatorname{WRIE}\left(*,{ }^{\prime}(\mathrm{i} 4, \mathrm{f} 10.3)^{\prime}\right) \quad \mathrm{i}, \mathrm{r}$

END PROGRAM mlp0

Listing 9: mlp2a.f90

SUBROUTINE init_name ()

COMMON /name/ i, r

$\mathrm{i}=786$

$\mathrm{r}=3.2$

REIURN

END SUBROUTINE init_name

If we only wish to use the routine init_name from $\mathrm{C}$ it is quite simple, we just modify init_name to the routine init_read as shown in Listing 10 and use the $\mathrm{C}$ program, given in Listing 11 to obtain the same result.

Listing 10: mlp2b.f90

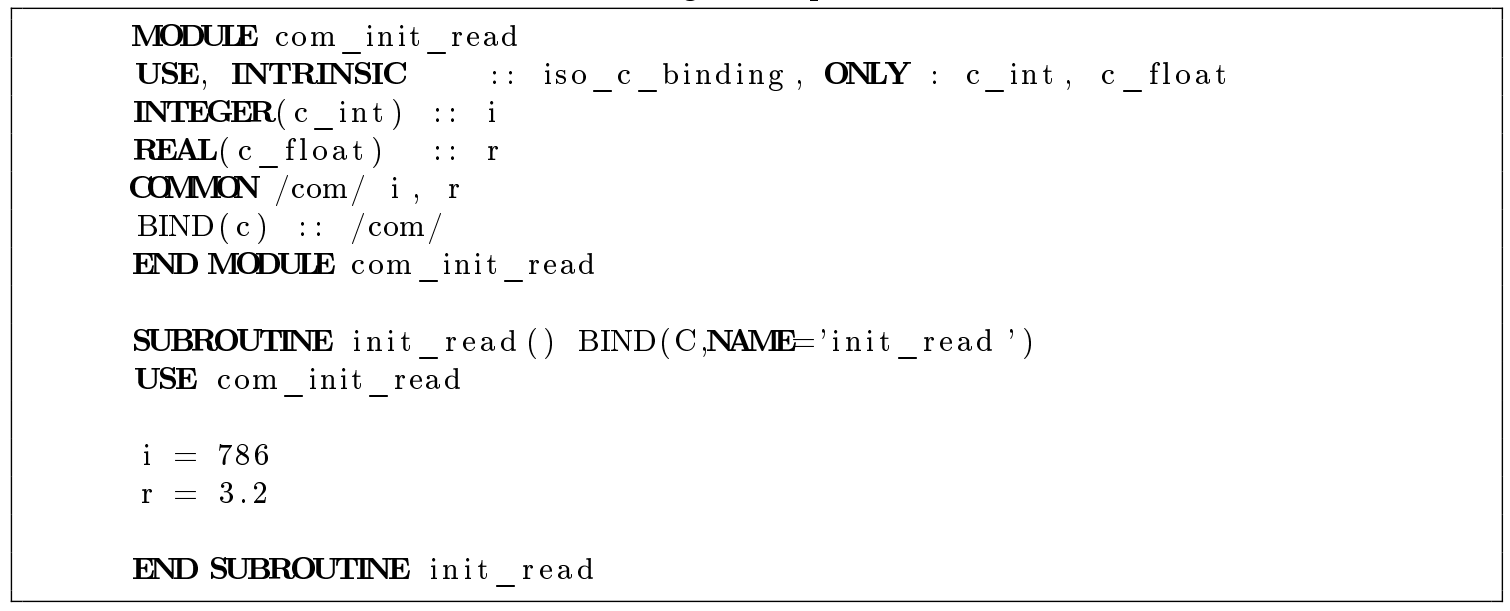

Listing 11: mlp1.c

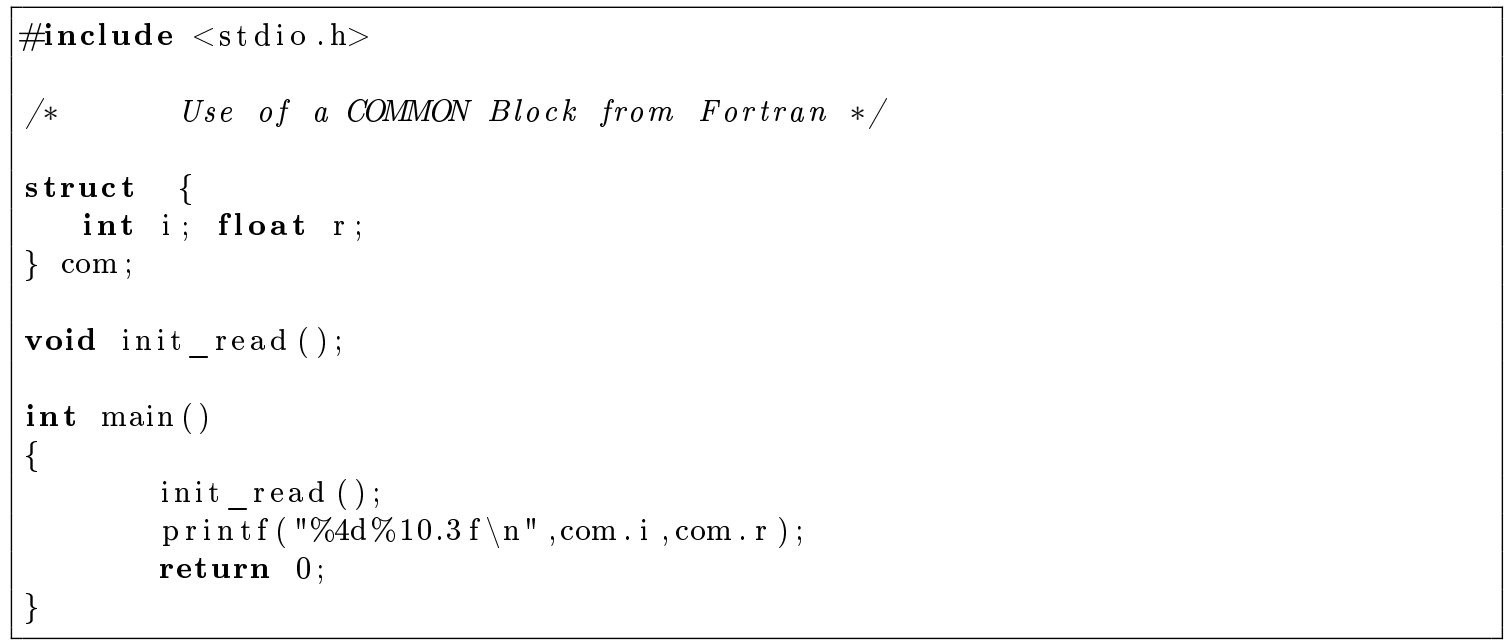

However the standard requires that if the BIND attribute is associated with one instance of a common block, it must be associated with all instances of that common block throughout the 
code. Thus, if we do not have access to the complete source code (e.g., we only have a precompiled library) providing the BIND attribute to a single definition of a common block that is used elsewhere will result in a non-conforming program. Even if the source code is available the changes required can be both numerous and error prone.

We therefore consider a second approach which uses a pointer and makes use of the fact that data stored in a common block must occupy a contiguous block of memory, see (Adams et al., 2008, section 5.14.2). We note here that this approach works best when all occurrences of the same common block have the same data layout.

The $\mathrm{C}$ routine is given in Listing 13 with the Fortran driver in Listing 12. Here we also let the $\mathrm{C}$ routine change the values in the COMMON block. This program may be compiled using

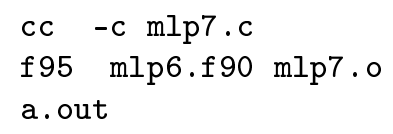

Listing 12: mlp6.f90

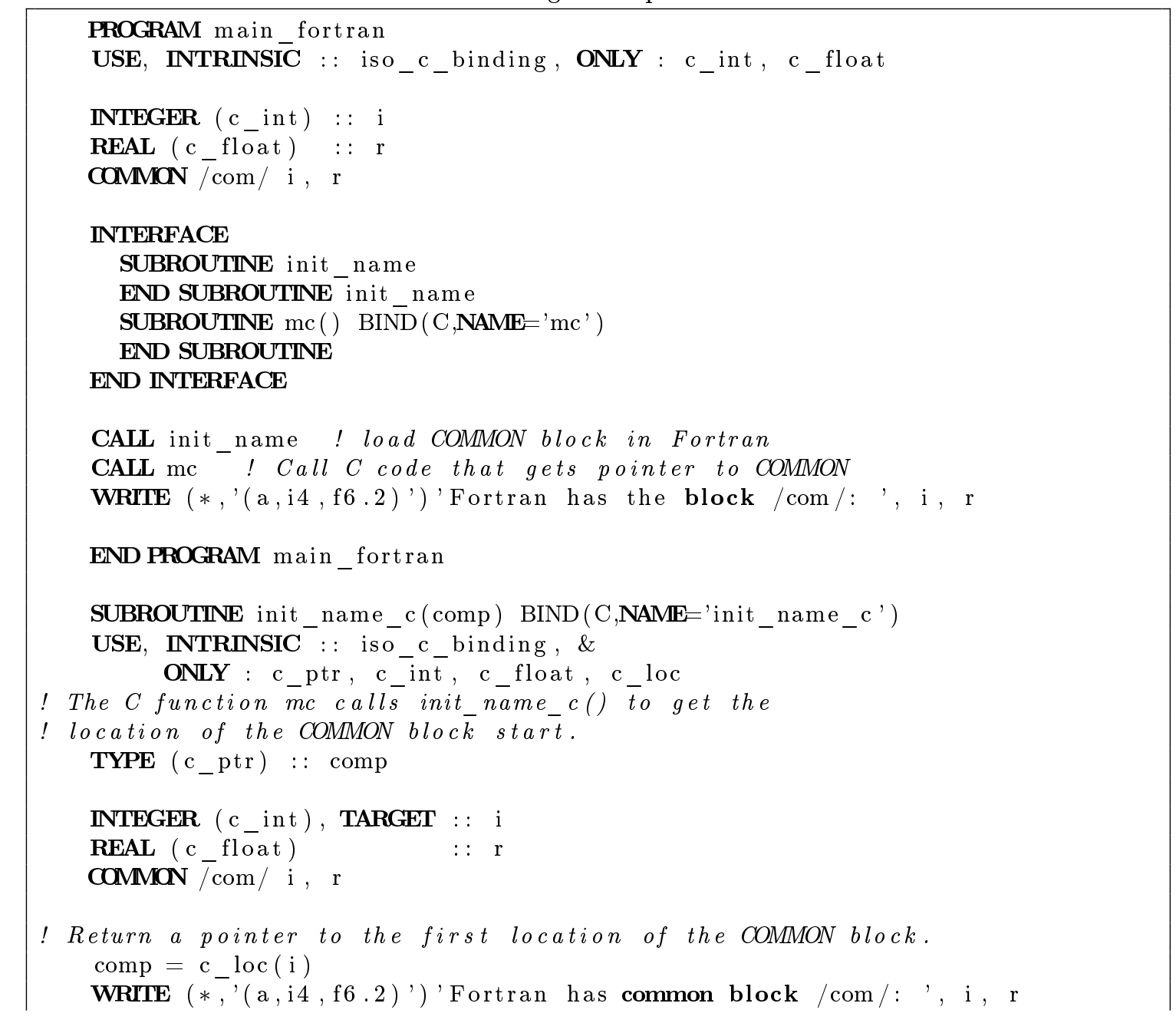


END SUBROUTINE init_name_c

SUBROUTINE init_name

! This is the original Fortran function

! assigning values to the COMMON BLOCK

INTEGER : : i

REAL : : $r$

COMMON / com/ i , r

$\mathrm{i}=786$

$\mathrm{r}=3.2$

END SUBROUTINE init_name

Listing 13: mlp7.f90

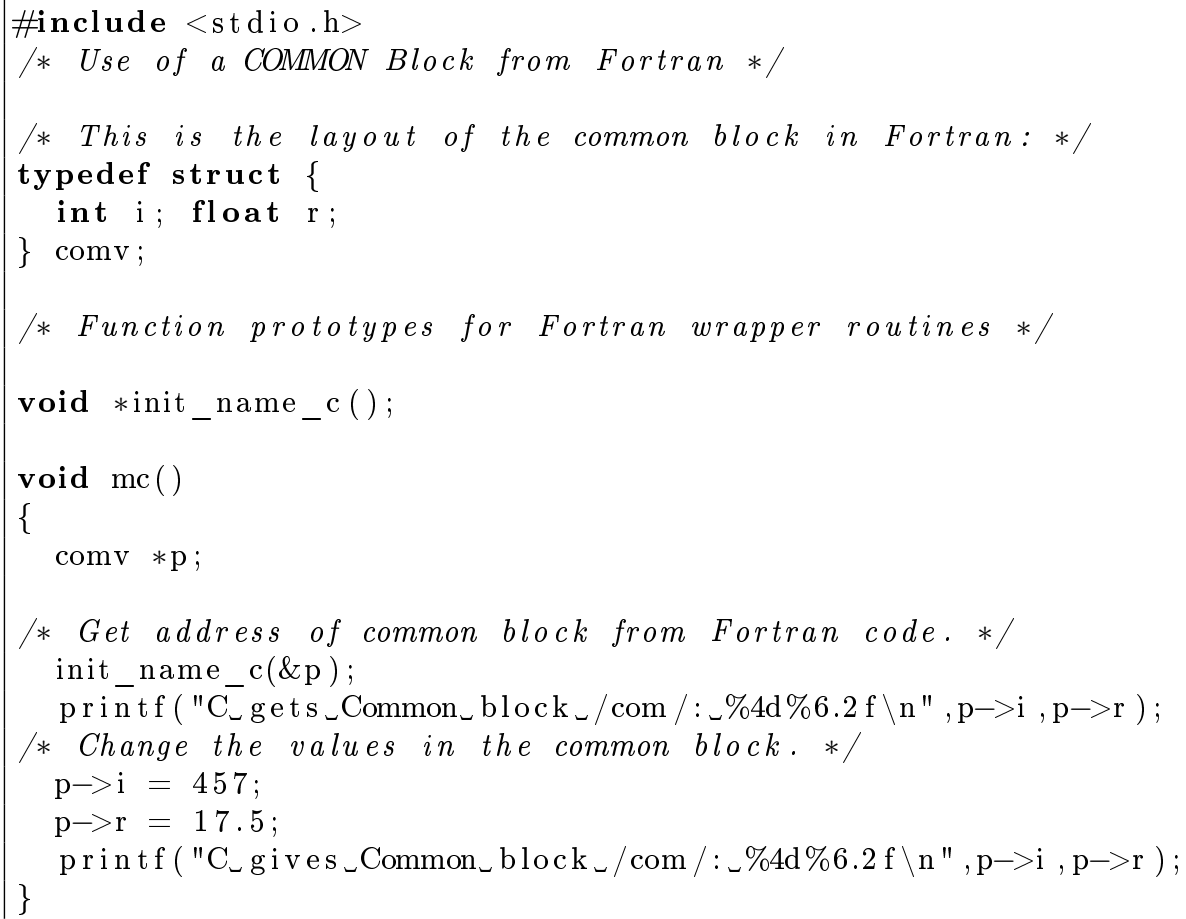

\section{Use of a C routine from Fortran}

As in the example above we wish to use a routine in $\mathrm{C}$ from a main program in Fortran, but in this case the $\mathrm{C}$ routine is a simple function.

As a simple example we write a function in C (see Listing 14) to evaluate the mean value of its two arguments. This may be called via the main program in Listing 15.

Listing 14: mean.c

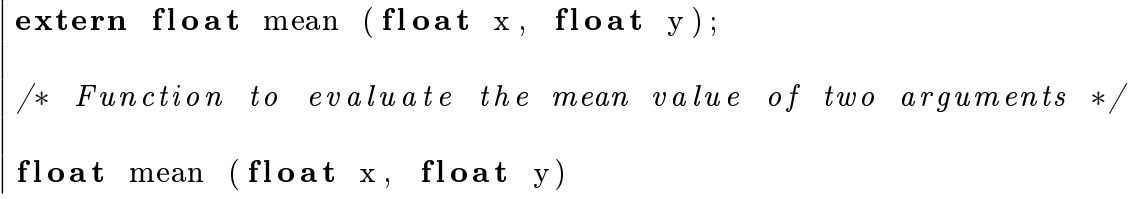


return $((x+y) / 2)$;

Listing 15: c_mean.c

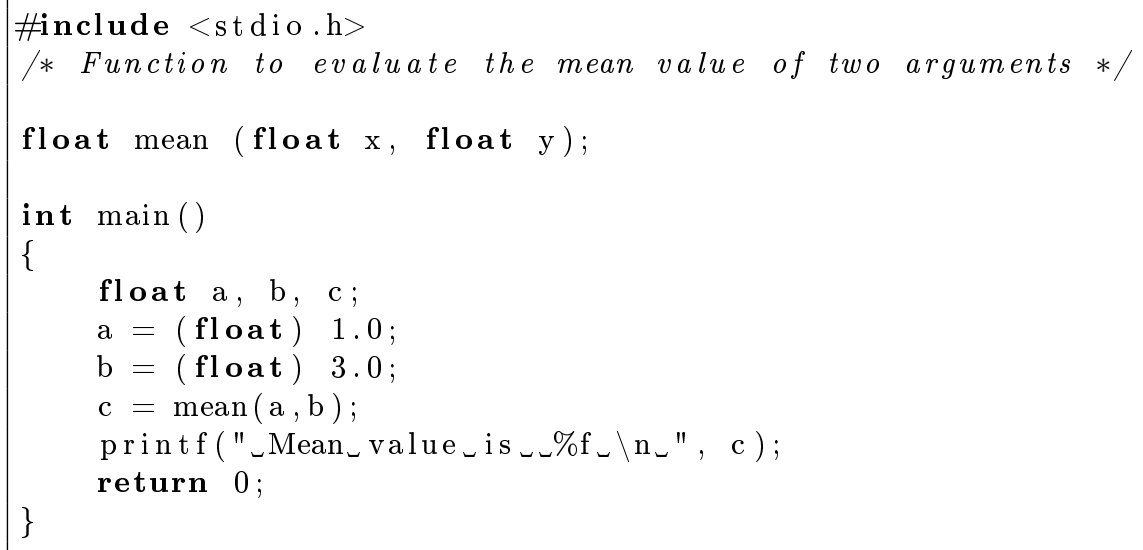

When a procedure is called in Fortran, by default, all arguments are passed by reference; i.e., the address of the argument is passed through to the routine. In $\mathrm{C}$ it is possible to pass arguments either by value or by reference; the cases are distinguished by passing a variable or a pointer to a variable of the relevant type respectively.

To allow Fortran to interoperate successfully with $\mathrm{C}$ it is necessary to be able to distinguish between these two types of argument. The VALUE attribute, introduced in Fortran 2003, enables procedure arguments to be declared as called by value. This facility is available for defining the way in which parameters are passed among Fortran procedures as well as for interoperability with C.

In the function mean the two parameters, $\mathrm{x}$ and $\mathrm{y}$, are both passed by value and hence, in the Fortran interface, these variables need to be declared with the value attribute. Failure to do this will result in the values being treated as addresses and either incorrect results or a fatal run-time error will result.

The complete program is provided in Listing 16.

Listing 16: $\operatorname{mlp} 8 . f 90$

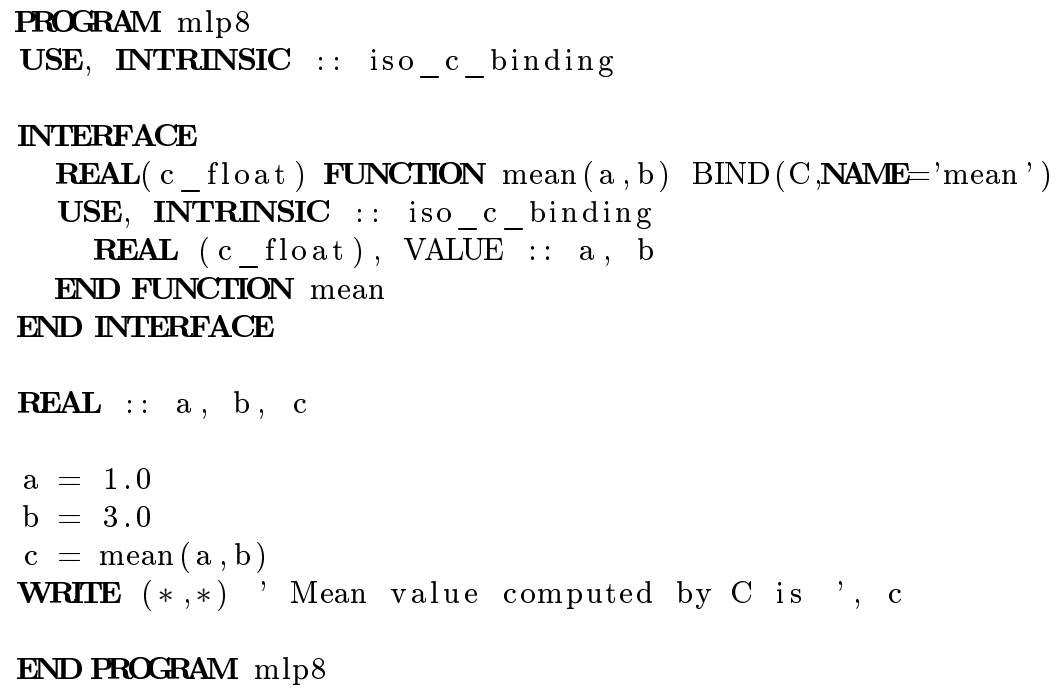




\section{GPU implementation}

This example is compelling because the topic of using an attached Graphics Processing Unit (GPU) is now under intense discussion in the High Performance Computing (HPC) community. Some attractive benchmarking results (Barrachina et al., 2008) have been obtained from using an NVIDIA chip with BLAS (Dongarra et al., 1990). These routines are the basics of the LAPACK (Anderson et al., 1999) package.

We use a version of the matrix multiplication routine SGEMM that uses a GPU implementation of a routine available in the NVIDIA CUBLAS library (CUDA, 2008).

CUDA is a general purpose parallel computing architecture introduced by NVIDIA that enables the GPU to solve complex computational problems. It includes the CUDA Instruction Set Architecture (ISA) and the parallel compute engine in the GPU. To program to the CUDA architecture, developers can use $\mathrm{C}$, which can then be run with great performance on a CUDA enabled processor. Other languages will be supported in the future, including Fortran and $\mathrm{C}++$.

This example is noteworthy for three reasons:

1. The use of this level 3 BLAS code is well-known to be one of the most important for numerical linear algebra. This particular GPU provides significant performance increases. At first glance it appears that one must replace calls to SGEMM with calls to cublasSgemm, the NVIDIA version. This can be avoided and is important when dealing with a pre-compiled application that uses SGEMM.

2. The NVIDIA documentation (CUDA, 2008) makes a statement that there is no standard interface for calling $\mathrm{C}$ functions from Fortran. The Fortran $2003 \mathrm{C}$ interoperability facilities provide a solution to this issue.

3. Using a replacement code for SGEMM that turns around and calls the NVIDIA code is easy to write in a standard way. As we have preserved the BLAS name using this approach we need to ensure that the correct version of SGEMM is linked into the final executable rather than a 'standard' version that may be included in one of the standard pre-compiled libraries our application uses. Placing the required code ahead of any such libraries in the link command is usually enough to achieve this.

The example here uses the VALUE attribute for some scalar arguments and also transfers Fortran CHARACTER data to $\mathrm{C}$ char data which we note are single characters in the $\mathrm{C}$ specification. We include below a version of the SGEMM routine. On the web site we also have a small driver program sgemm_driver. $\mathbf{9} 90$ (also with explanation of all the arguments) and a C dummy routine c_sgemm.c. Our intent is to emulate the interface to the C NVIDIA code by using an identical specification for the arguments. NVIDIA wrote the $\mathrm{C}$ code to have column-oriented storage!

Listing 17: sgemm.f90

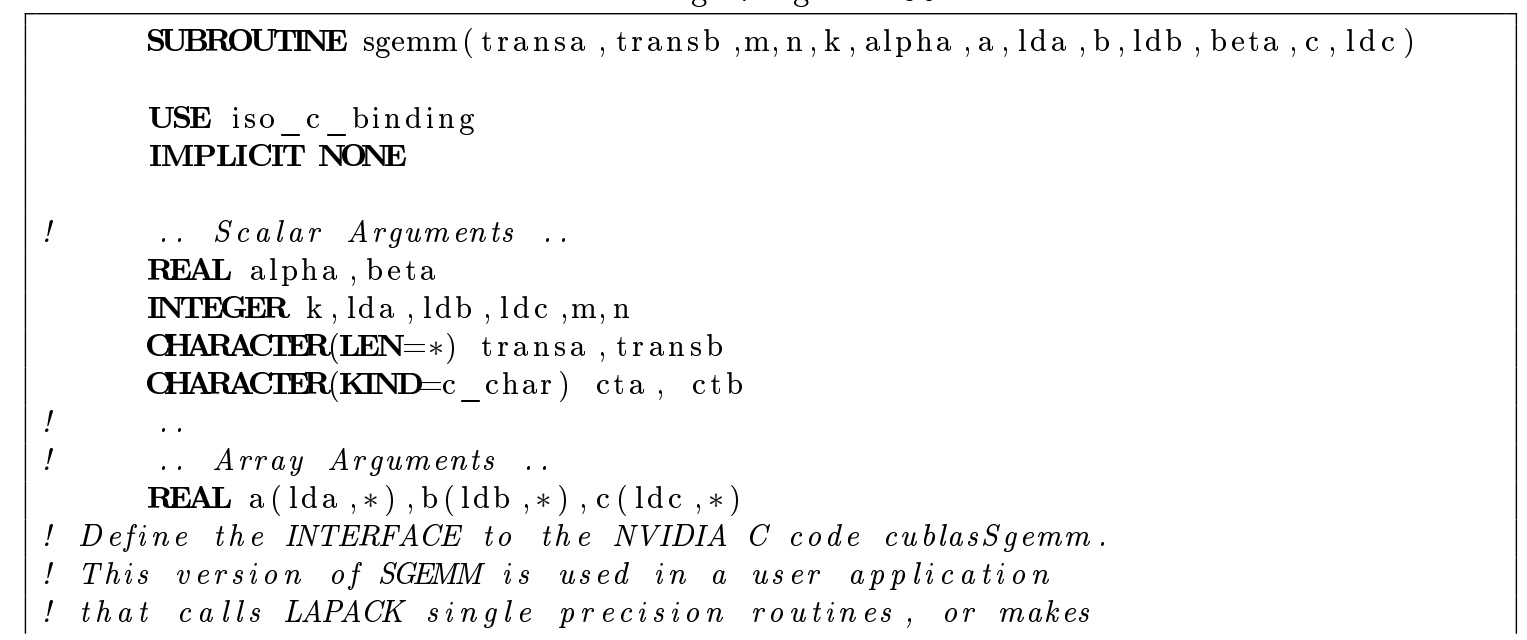




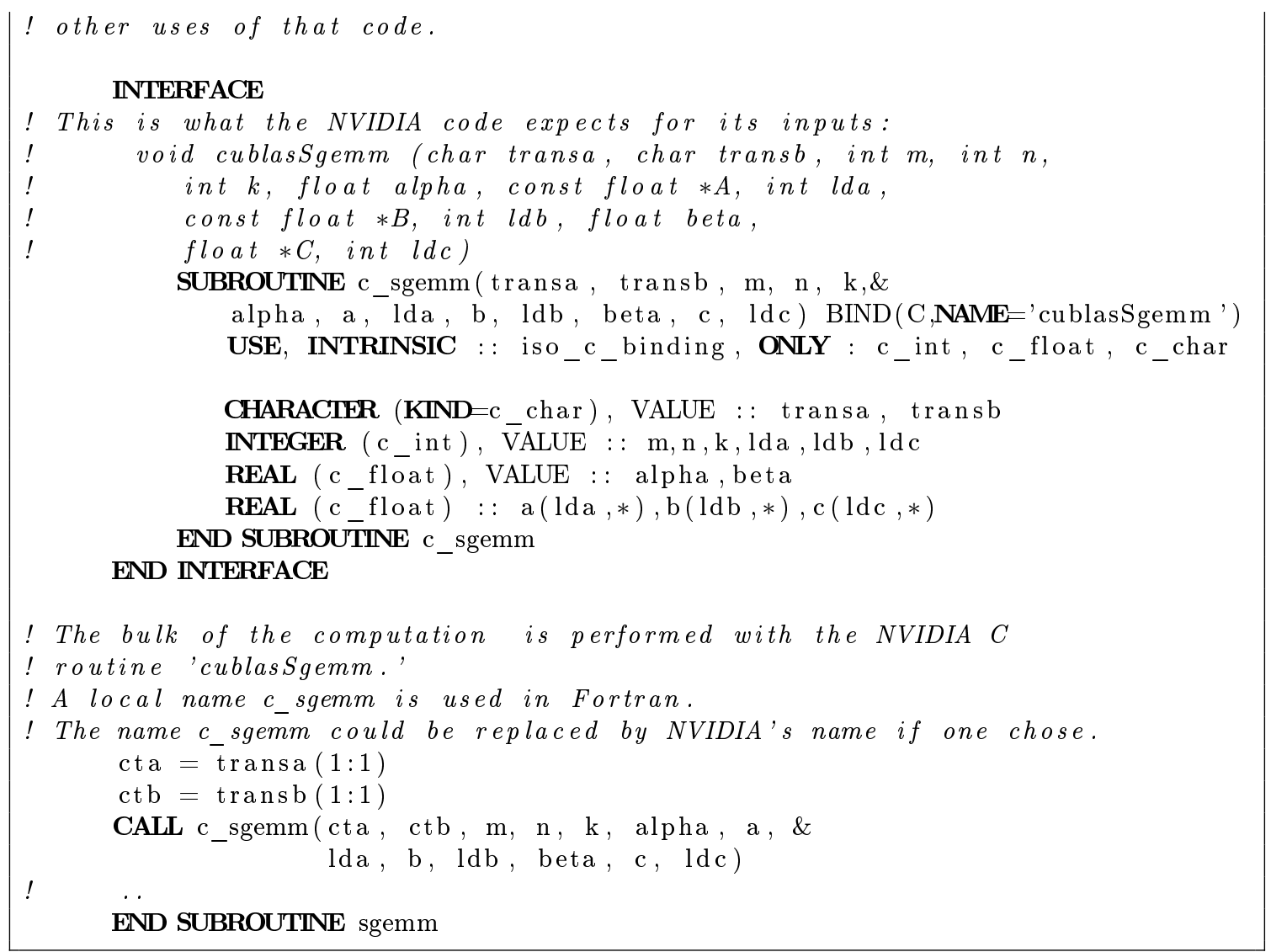

In practice, to ensure maximum efficiency, the GPU would only be used when the problem size exceeded some break-even point; smaller problems would be dealt with directly on the master CPU, for example, using either the Intel MKL (Intel, 2008) or the vanilla code available from netlib (http://www.netlib.org/blas/). The break-even point is dependent on the GPU device being used and benchmarking would be needed to determine a suitable value.

The character arguments to sgemm are declared to be CHARACTER (LEN=1) in the definition of the routine in (Dongarra et al., 1990). It appears to be almost standard practice to use longer, more descriptive, constant, actual arguments; for example, 'TRANSPOSE' rather than 'T'. However, the Fortran standard states that only the first character of such arguments is used and thus the arguments to sgemm can be passed directly to c_sgemm provided that the default character type is interoperable. However, this does not work with all compilers, hence the use of the extra variables cta and ctb.

\section{Enumerated Types}

Fortran 2003 introduced a new enumeration definition mainly to allow interoperability with enumeration constants in $\mathrm{C}$ although it may be used freely within Fortran code. The standard guarantees that constants declared as type enumerator will correspond to the same integer type used by C, i.e., int.

An example of an enumeration in Fortran is shown in Listing 18 and is very similar to the enum definition in $\mathrm{C}$.

Listing 18: Enumerator Example

ENUM, BIND $(\mathrm{C})$

ENUMERATOR : : jan $=1$, feb, mar, apr, may, jun, \& jul, aug, sep, oct, nov, dec 
An enumerator constant may either be initialized explicitly (as jan above) or implicitly when it takes a value one greater that the previous enumerator constant in the list. If the first enumerator constant in the list is not explicitly initialized it is set to zero.

The Fortran standard provides no means of directly determining the kind selected for the integer used to store an enumerator value. Variables that may be assigned enumerator constants therefore need to be declared as, for example,

$$
\text { INTEGER(KIND( jan )) : : month }
$$

The simple Fortran subroutine and driver program given in Listings 19 and 20 illustrate how the enumerator constants are used completely within Fortran.

Running this code gives the output

month 7 has 31 days

month 2 has 28 or 29 days

Listing 19: f_print_days.f90

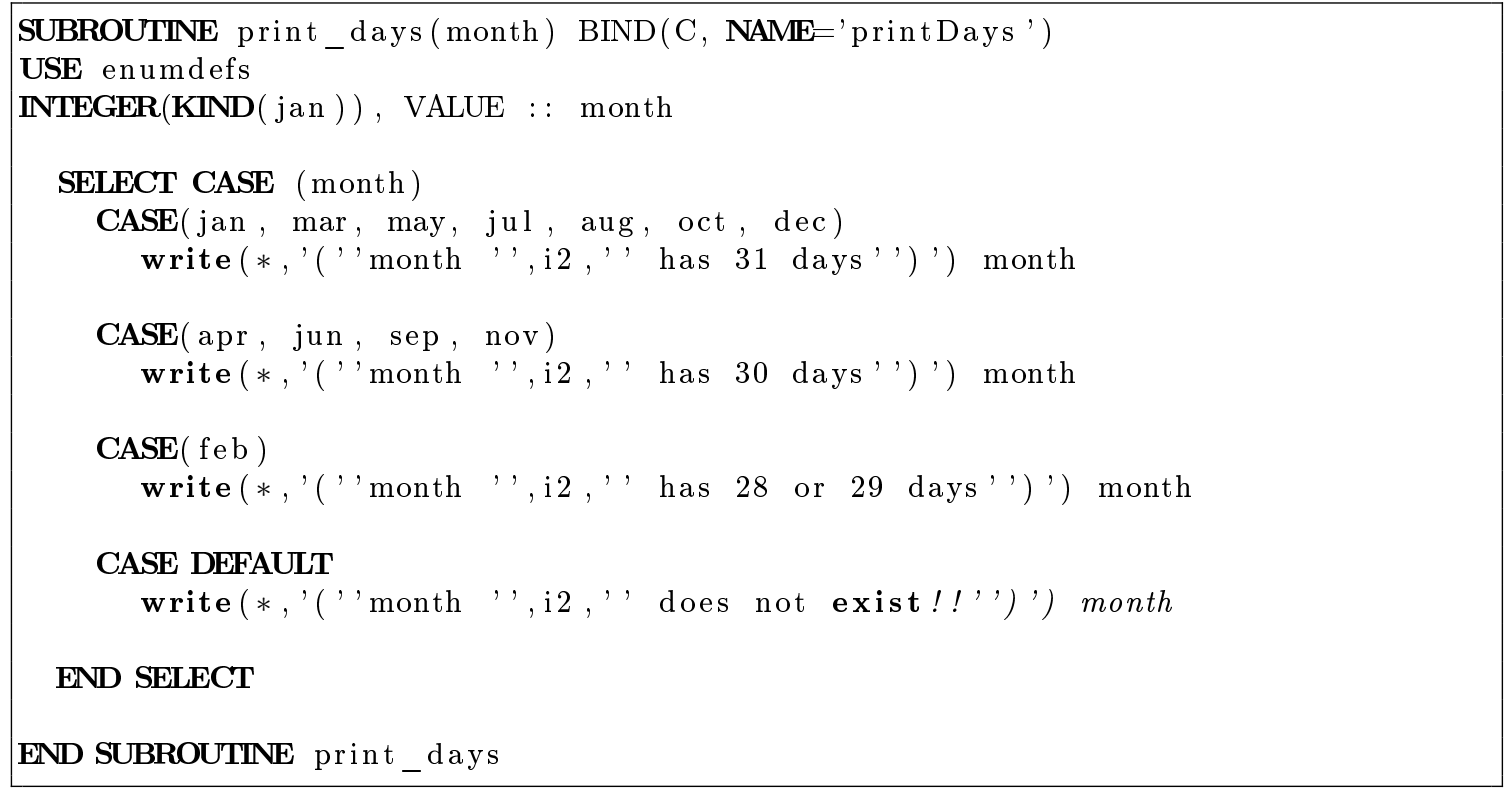

Listing 20: f main.f90

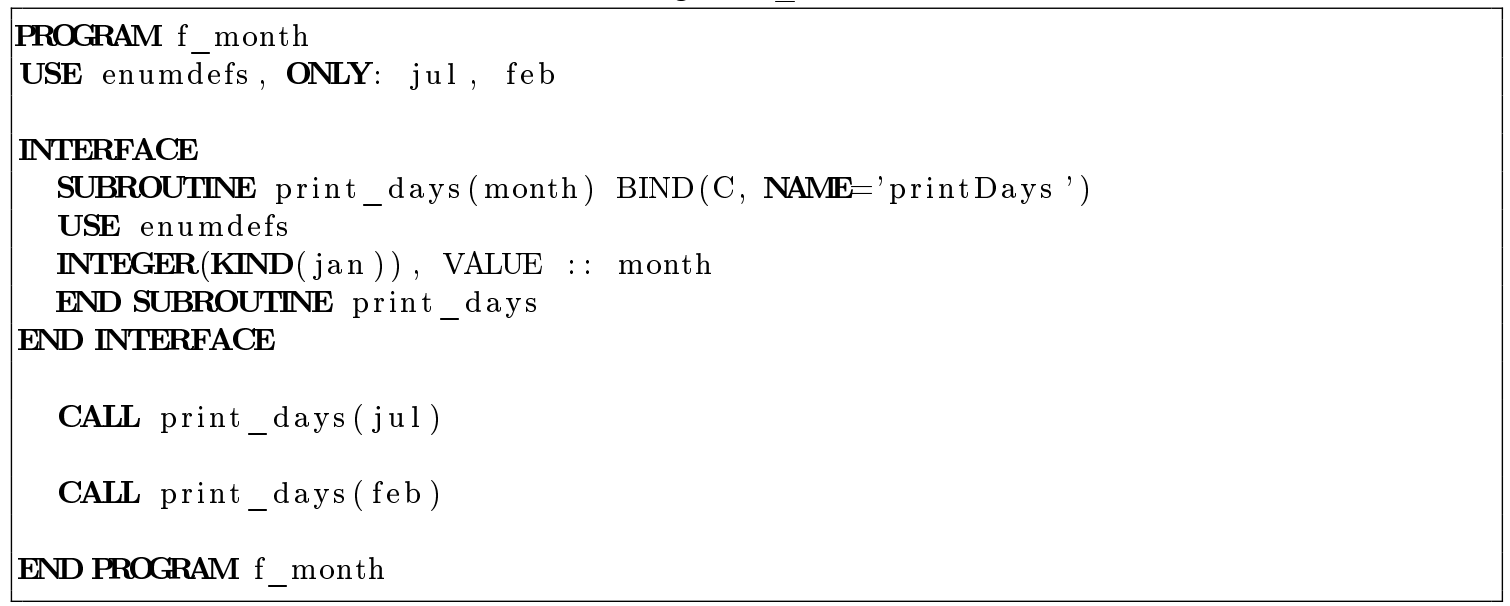


Listing 21: c_print_days.c

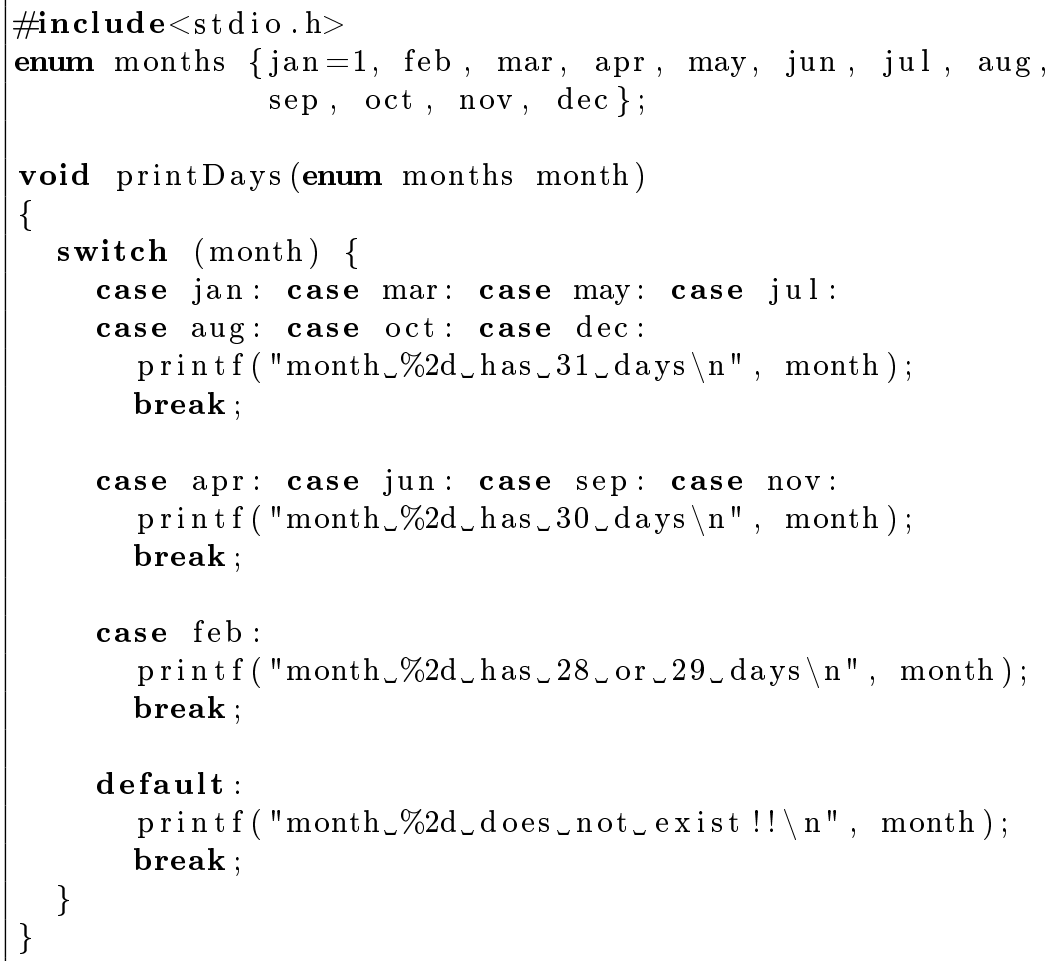

Combining $\mathrm{f}$ _main and the $\mathrm{C}$ version of print_days given in Listing 21 illustrates how enumeration constants interoperate.

Linking the Fortran version of print_days with the $\mathrm{C}$ driver program in Listing 22 provides a second example.

Listing 22: c_main.c

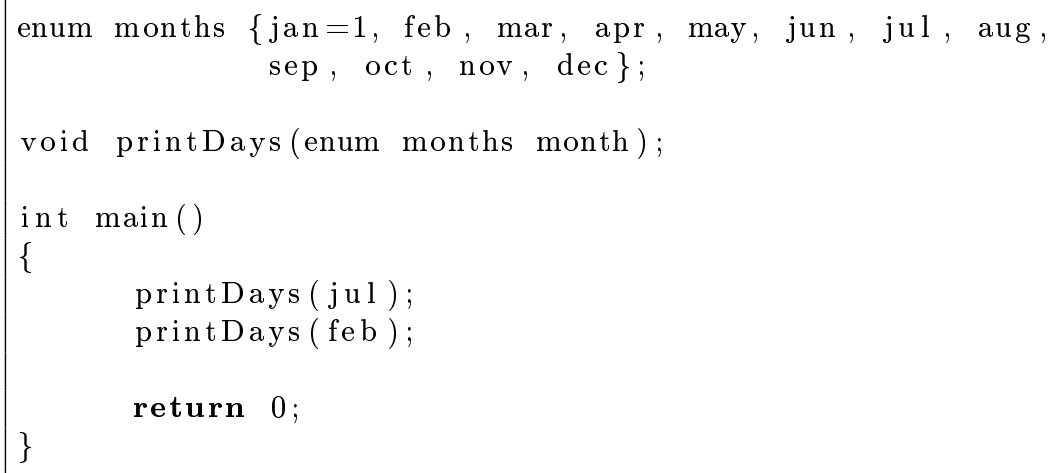

\section{Other Functionality}

We finish by mentioning a number of additional features and functionality that the iso_c_binding module provides that we have not mentioned.

"Pointers" for $\mathrm{C}$ and Fortran are very different and are not interoperable with one another. A C pointer is just an address and, since it is considered to be a separate type, can be used to point to data of any type. On the other hand a Fortran pointer is defined to be of the same type as its target. Second, when considering array data, a Fortran pointer needs to store additional information regarding the bounds and shape of the array making it incompatible with C's concept 
of a simple address in memory. For example, it is not possible to make a non-contiguous array section the target of a $\mathrm{C}$ pointer. However, non-contiguous array sections can still be passed as actual arguments to an interoperable $\mathrm{C}$ function since Fortran will perform copy-in and copy-out under these circumstances.

The iso_c_binding module provides two derived types to allow interoperability with $\mathrm{C}$ data pointers (c_ptr) and $\mathrm{C}$ function pointers (c_funptr). These may be used, for example, to preserve access to non-interoperable $\mathrm{C}$ data that needs to be preserved between calls to $\mathrm{C}$ functions. Accompanying these derived types are five subroutines and inquiry functions, (c_loc, c_funloc, c_associated, c_f_pointer, c_f_procpointer) that allow a variety of tests and conversions to be performed. For full details of the rules and restrictions governing these functions we refer the reader to (Adams et al., 2008, pp.569-579).

\section{Closing Remarks}

All the files mentioned here are available for testing, together with explanations on how to compile them, at http://www.nsc.liu.se/wg25/mlpcode/.

We also tested our examples using Sun Fortran 958.2 (October 13, 2005). There is a minor error in this implementation; the standard requires

$$
\text { subroutine } \operatorname{sub}(f, b) \text { bind(c) }
$$

but this has to be written, using this version of the Sun compiler with a comma in order to avoid compilation errors

$$
\text { subroutine } \operatorname{sub}(f, b) \text {, bind(c) }
$$

A similar remark holds for functions. This comma is optional in Sun Fortran 958.3 (July 18, 2007).

There is also a problem on some systems in using CHARACTER (c_char) since CHARACTER (length) has the same syntax. We therefore recommend using CHARACTER (KIND=c_char).

The interoperability problem can be messy, even with the standard. For example, the enum construct (Metcalf et al., 2004, Section 14.11) is not implemented in all compilers. It is, for example, on IBM but not Intel $10.1^{3}$, Sun 8.3, and Portland pgf90 8.0-5. Not only that, but Microsoft $\mathrm{C}++$ does not have Bool as a supported type. This makes portability difficult! If a programmer has to deal with problems like these then one may have to write two wrappers - in both languages to deal with non-portable data types and constructs, but the two wrappers can have a standard interface.

\section{Acknowledgements}

We wish to thank Jack Dongarra, University of Tennessee, for giving us access to a system with the NVIDIA processor. Massimiliano Fatica at NVIDIA illustrated the memory management utilities for getting SGEMM to work from Fortran. Pär Andersson of the National Supercomputer Centre in Sweden made the tests with Intel 11. W. Van Snyder, of JPL and a member of IFIP WG2.5, provided insight regarding the Fortran 20003 and previous standards.

\section{References}

Adams, J. C., Brainerd, W. S., Hendrickson, R. A., Maine, R. E., Martin, J. T., and Smith, B. T. (2008). The Fortran 2003 Handbook: The Complete Syntax, Features and Procedures. Springer, London. ISBN 978-1-84628-378-9.

\footnotetext{
${ }^{3}$ It is confirmed that it is available on Intel 11.0.074.
} 
Anderson, E., Bai, Z., Bischof, C., Blackford, S., Demmel, J., Dongarra, J., Du Croz, J., Greenbaum, A., Hammarling, S., McKenney, A., and Sorensen, D. (1999). LAPACK Users' Guide. SIAM, Philadelphia, third edition. ISBN 0-89871-447-8.

Barrachina, S., Castillo, M., Iqual, F. D., Mayo, R., and Quintana-Orti, E. S. (2008). Evaluation and tuning of the Level 3 CUBLAS for graphics processors. In Proceedings of 22nd IEEE International Symposium on Parallel and Distributed Processing, IPDPS 2008. Miami, Florida USA, April 14-18, 2008, pages 3:1-8, IEEE, Los Alamitos, CA, USA. ISBN 978-1-4244-1693-6.

Chivers, I. D. and Sleightholme, J. (2009). Compiler support for the Fortran 2003 standard. ACM SIGPLAN Fortran Forum, 28(1):26-28. ISSN:1061-7264.

CUDA (2008). CUBLAS Library. Technical Report PG-00000-002_V2.0, NVIDIA, Santa Clara, CA 95050.

http://developer.download.nvidia.com/compute/cuda/2_0/docs/ CUBLAS_Library_2.0.pdf.

Dongarra, J., Du Croz, J., Duff, I., and Hammarling, S. (1990). A set of level 3 Basic Linear Algebra Subprograms. ACM Trans. Math. Soft., 16(1):1-17.

Einarsson, B. (1995). Mixed Language Programming, Part 4, Mixing ANSI-C with Fortran 77 or Fortran 90; Portability or Transportability? In Current Directions in Numerical Software and High Performance Computing, International Workshop, Kyoto, Japan. IFIP WG 2.5. http://www.nsc.liu.se/ boein/ifip/kyoto/einarsson.html.

Intel (2008). Intel@Math Kernal Library. Santa Clara, CA, USA, 630813-029US edition.

Metcalf, M., Reid, J., and Cohen, M. (2004). Fortran 95/2003 explained. Numerical Mathematics and Scientific Computation. Oxford University Press. ISBN 0-19-852693-8. 